\title{
INVESTIGACIÓN
}

\section{CONTRIBUCION AL CONOCIMIENTO DE ESPECIES APIBOTÁNICAS EN DOS REGIONES DE MEZQUITAL, DGO., MEX.}

\author{
Contribution to species knowledge \\ apibotanics in two regions of Mezquital, Dgo., Mex.
}

EPISTEMUS

ISSN: 2007-8196 (electrónico)

ISSN: 2007-4530 (impresa)

David Ramírez Noya ${ }^{1}$

María P. González Castillo 2

Recibido: 14 de octubre de 2016,

Aceptado: 13 de noviembre de 2016

Autor de Correspondencia:

M. en C. DAVID RAMÍREZ NOYA

Correo:davidrnoya@yahoo.com.mx

\section{Resumen}

Se reportan 115 especies, en 98 géneros de 38 familias botánicas que son fuente de alimento para las abejas (Apis mellifera L.), presentes en dos regiones del municipio del Mezquital, Dgo. y una descripción breve del tipo de vegetación en relación a las especies dominantes, en las localidades donde se realizó el estudio.

Palabras clave: Flora apícola, bosque tropical caducifolio.

\section{Abstract}

A preliminary checklist of 115 species (belonging to 98 genera and 38 families) like food resource (nectar and/or pollen) for honey bee (Apis mellifera $L$.) of two regions of Durango state, is presented. And dominants species in vegetation communities presents in studio area, are cited.

Keywords: apibotanics species, tropical deciduous forest.

1 Departamento de Gestión de Proyectos, Universidad de las Ciencias Informáticas. \{abp, robertodv\}@uci.cu

2 Facultad de Ingeniería, Universidad Católica Santiago de Guayaquil, Ecuador. gilberto.castro@cu.ucsg.edu.ec 


\section{INTRODUCCIÓN}

La exploración botánica en el área geográfica del Mezquital, Dgo., se inician en el siglo XIX con el recorrido realizado por Berthold Carl Seemann, en enero de 1850, en su trayecto de la cd de Durango hacia el Estado de Nayarit. Posteriormente J. N. Rose y E. A. Goldman el año de 1897, hacen recolecciones principalmente en los alrededores de Huazamota, Mezquital, Dgo. [1]. Pero los estudios respecto a plantas de importancia para Apis mellifera L., en el estado de Durango, inician hasta mediados del siglo XX [2]. Siendo más específico, para el municipio de Mezquital, Dgo., los trabajos referentes a la flora de importancia apícola, se reinician en años recientes [3], incluyendo análisis polínico en muestras de miel para hacer una caracterización de las mismas [4]; además se aplica un análisis de la composición química del polen de las especies apibotánicas, con el fin de determinar, según la especie vegetal, el valor nutricional que representa para las abejas [5]; por otra parte se aplican los sistemas de información geográfica, con el fin de hacer una representación espacial de las especies pertenecientes a las dos familias de fanerógamas más importantes para la apicultura [6]. Finalmente, mediante un análisis ecológico estima la diversidad de árboles y arbustos que son fuente de alimento para las abejas, en dos localidades del municipio del Mezquital, Dgo. [7]. Por lo que en el presente trabajo se pretende dar continuidad al conocimiento de la diversidad de los recursos néctar-polínicos utilizados por Apis mellifera, en el Mezquital, Durango.

El municipio del Mezquital está ubicado en el extremo sur del estado de Durango, su extensión territorial es de 8,430 kilómetros cuadrados. Limita al sur, con el estado de Nayarit; al este, con el estado de Zacatecas; al norte, con los municipios de Súchil, Nombre de Dios y Durango; y al poniente con el municipio de Pueblo Nuevo [8]. La orografía del Mezquital es intrincada y accidentada, en su extenso territorio se pueden distinguir tres diferentes zonas, la del extremo norte donde se encuentra valles que descienden desde la altas serranías, la región media, totalmente ocupada por la Sierra Madre Occidental, y finalmente la región de las quebradas, formada por los abruptos descensos de la Sierra hacia las llanuras costeras del Pacífico, en esta zona destaca la Cañada del río Huazamota [9]. La máxima altitud del estado se localiza dentro del municipio del Mezquital, el cerro Gordo con 3340msnm [10].

La extensión territorial total, del municipio en estudio, da idea lo complejo que sería aplicar un análisis más completo, que permitiera obtener un conocimiento más íntegro sobre los recursos naturales que ostenta esta región del estado de Durango.

\section{OBJETIVO}

Contribuir al conocimiento de la diversidad de los recursos néctar-polínicos utilizados por Apis mellifera, en el Mezquital, Durango.

\section{MÉTODO DE TRABAJO}

Para el muestreo de los elementos de la vegetación y el registro fotográfico de las especies de importancia a las abejas, se hicieron recorridos tanto en las proximidades a la cabecera municipal del Mezquital, Dgo., como en las cercanías de la población de Temoaya, Mezquital, Dgo. Siendo éstas, las dos localidades que circunscribieron al presente estudio. Dichas poblaciones presentan las

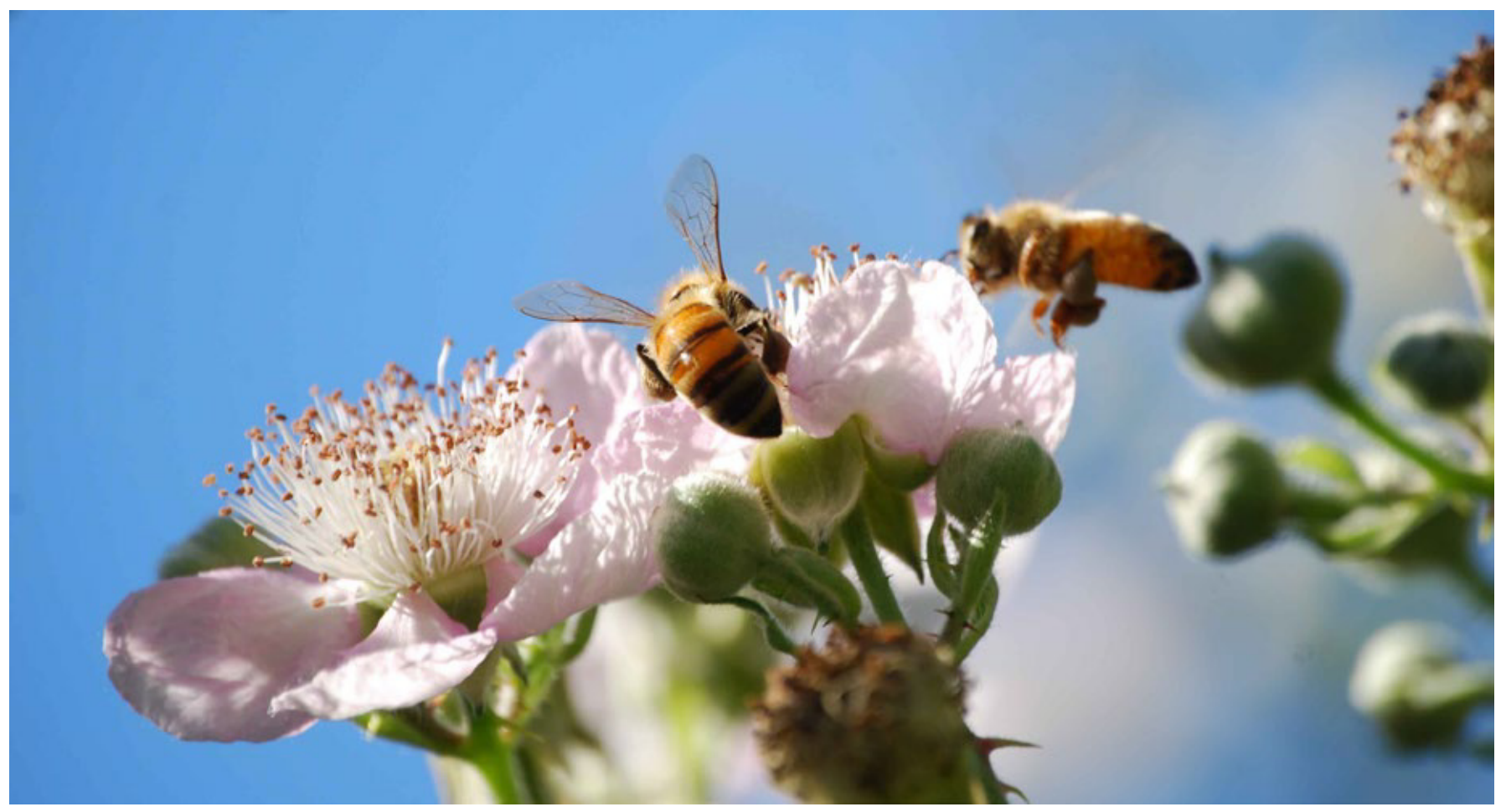


coordenadas $23^{\circ} 28^{\prime} 25^{\prime \prime} \mathrm{N}$. y $104^{\circ} 23^{\prime} 46^{\prime \prime}$ W.; $23^{\circ} 17^{\prime}$ $59^{\prime \prime}$ N. y $104^{\circ} 30^{\prime} 33^{\prime \prime}$ W., con altitudes de 1412 m. y 1292 msnm., respectivamente [11]. Ambas áreas geográficas del presente estudio presentan una temperatura media anual de entre 20 a $22^{\circ} \mathrm{C}[12]$ y poseen clima de tipo semicálido subhúmedo (ACw) [10].

Para la descripción de la vegetación de las localidades de estudio en el presente, se tomó como referencia la clasificación descrita por S. González; M. González y M. Márquez [10]; con esta base, durante los recorridos se detectaron áreas cuyos rasgos fisonómicos de las comunidades vegetales abarcaron desde, matorral subtropical (constituido básicamente por; Ipomoea arborescens, Acacia farnesiana, A. constricta, Bursera fagaroides, Dodonea viscosa y Lippia graveolens) hasta el bosque tropical caducifolio (donde los dominantes fisonómicos son esencialmente: Ipomoea murucoides, Ipomoea arborescens, Stenocereus montanus, Bursera fagaroides, B. multijuga, B. palmeri, Myrtyllocactus geometrizans, Acacia farnesiana, Prosopis laevigata, Agave vilmoriniana, Lonchocarpus sp y Lysiloma spp.). También se visitaron parte de los ecotonos de los anteriores tipos de vegetación, con el matorral xerófilo y con bosque de Quercus spp. Respecto a las exploraciones realizadas en las proximidades a Temoaya, la vegetación observada solo corresponde a lo aquí es señalado para el bosque tropical caducifolio.
Para la integración del listado de las especies vegetales, se auxilió atreves del registro fotográfico y la recolección e identificación taxonómica correspondiente a las plantas visitadas por estas abejas, por ser dichos vegetales fuentes de néctar ó polen. Estos transectos se aplicaron en las proximidades de ambas localidades arriba mencionadas, durante los años del 2016 al 2018; en las cuatro estaciones del año, con un total cercano a 65 recorridos. Para la determinación taxonómica de las especies de plantas registradas, se obtuvo mediante la técnica de herborizado descrita por A. Lot y F. Chiang [13]. Trabajando este material biológico en el Herbario CIIDIR del Instituto Politécnico Nacional, en Durango, Dgo.

\section{RESULTADOS}

Se estima que en el presente trabajo se exploró una superficie aproximada de $200 \mathrm{Km}^{2}$. Dicha estimación de área explorada representaría el $2.7 \%$ del total municipal. En consecuencia se obtuvo un listado de 115 especies en 38 familias (Listado 1). Aunque estos resultados son parciales, se vislumbra una significativa diversidad de especies (de la cual las abejas hacen uso del recurso), debido a que las cantidades aquí obtenidas, son comparativamente cifras superiores al contrastar con los números obtenidos en el Municipio de Vicente Guerrero, Dgo. [14]-[15], En el cual se adquirieron 132 especies en 32 familias, para una superficie mayor de exploración $\left(432 \mathrm{Km}^{2}\right)$.

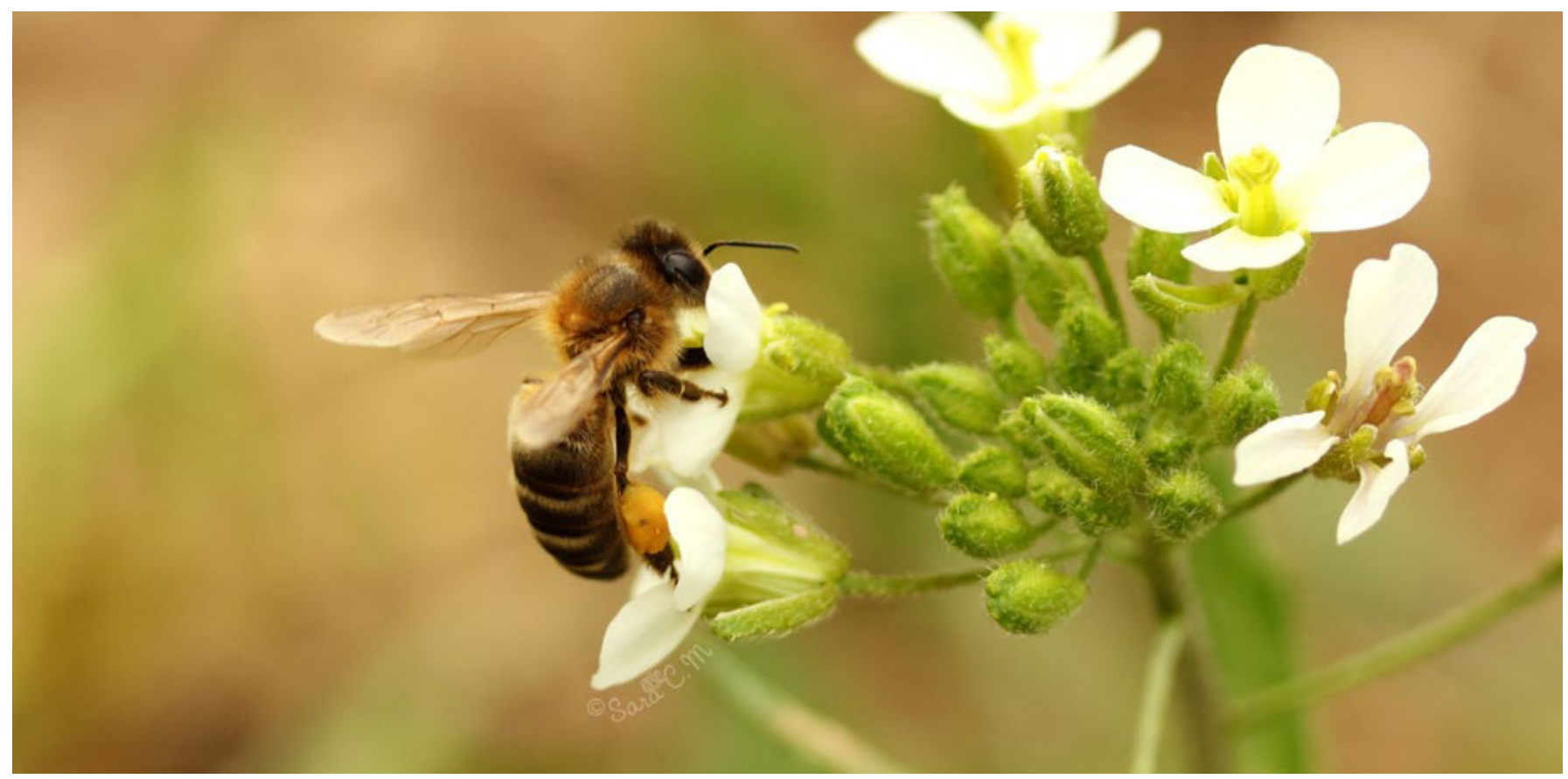




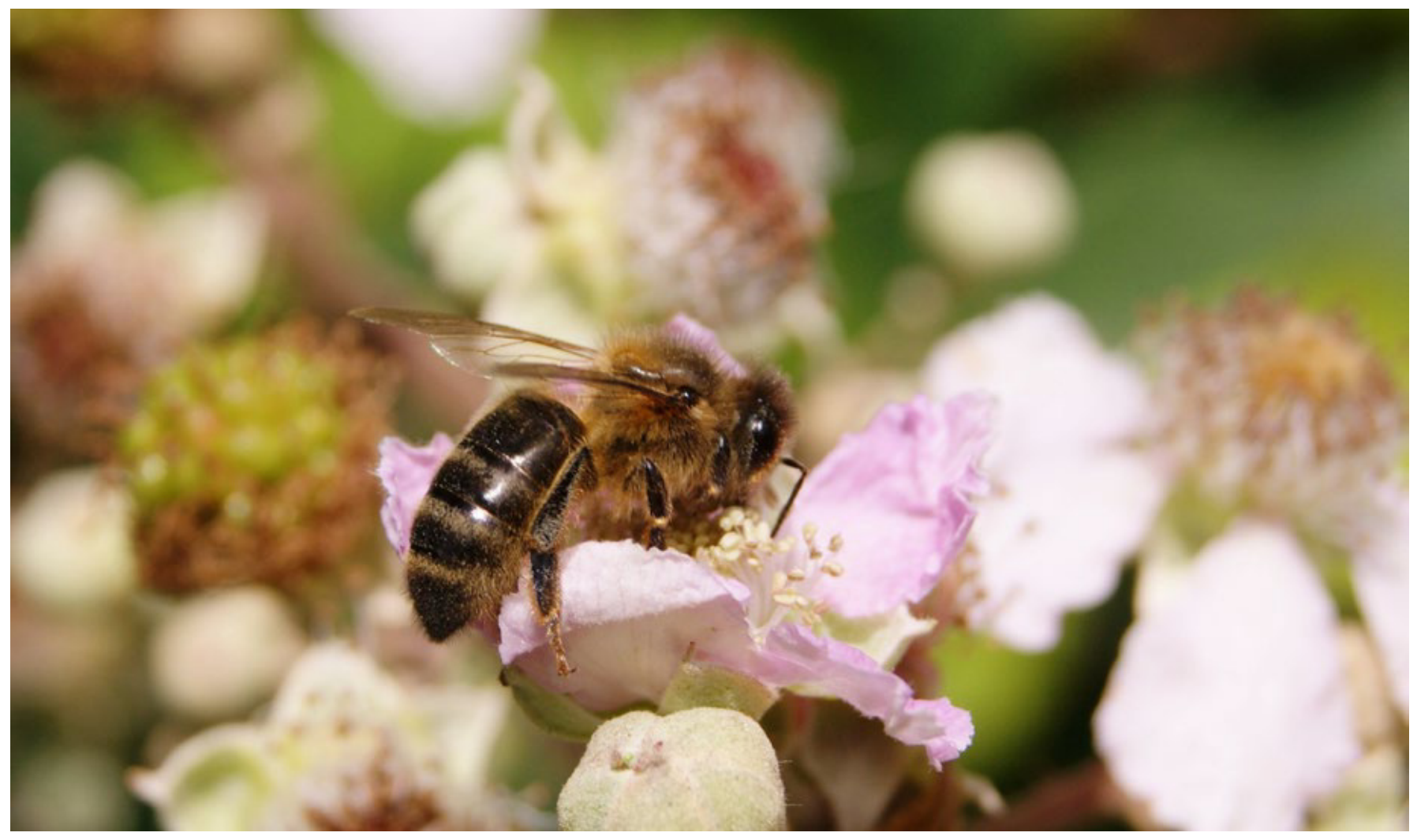

En el presente trabajo las familias botánicas de mayor importancia apícola fueron las Asteraceae (22.6\%) con 26 especies y Fabaceae (17.4\%) con 20 especies, mientras que 14 familias de plantas presentaron una especie.

Adicionalmente se presentan los nuevos registro de especies apibotánicas para el municipio del mezquital, Dgo. y que a su vez también son nuevos registros para la flora apícola del estado de Durango. (Listado 2).

\section{DISCUSIÓN}

Para un apicultor, el listado de especies vegetales aquí indicado, resulta interesante para valorar en cual región - localidad, ubicar o remover sus colmenas, por las característica de su diversidad vegetal, con el fin de obtener ganancias mayores, por el tipo de miel a cosechar (o producto apícola de interés), permitiéndole con esto elaborar su programa de actividades anual. En contraste, para un botánico; de dicho listado, lo importante será conocer, cuales son los nuevos registros de especies para Durango y su diversidad biológica.

\section{CONCLUSIÓN}

Los listado que aquí se presentan, es una versión en extenso, de los resultados ya reportados [3]-[4]-[6]-[7]-[16]. Además estos mismos listados de especies permitirán avanzar en la determinación de varios de los taxa al que pertenecen algunas de las muestras, del análisis palinológico [3]-[4], en las que está pendiente la definición del género o la especie correspondiente.

\section{AGRADECIMIENTOS}

Al Consejo de Ciencia y Tecnología del estado de Durango y al Instituto Politécnico Nacional. Proyecto: Caracterización polínica de mieles procedentes de los valles de Durango y el Mezquital, Durango, clave: SIP-2018-RE/039 y SIP-IPN: 20161358; por el apoyo financiero para la realización de los mismos.

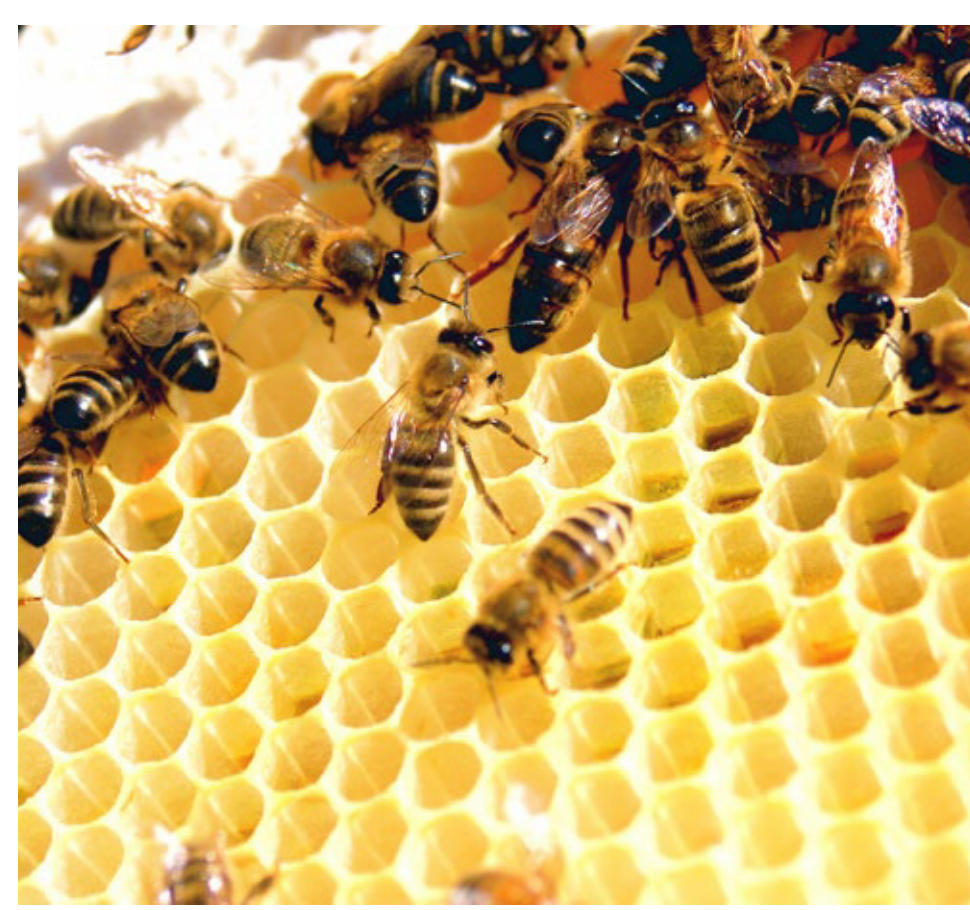



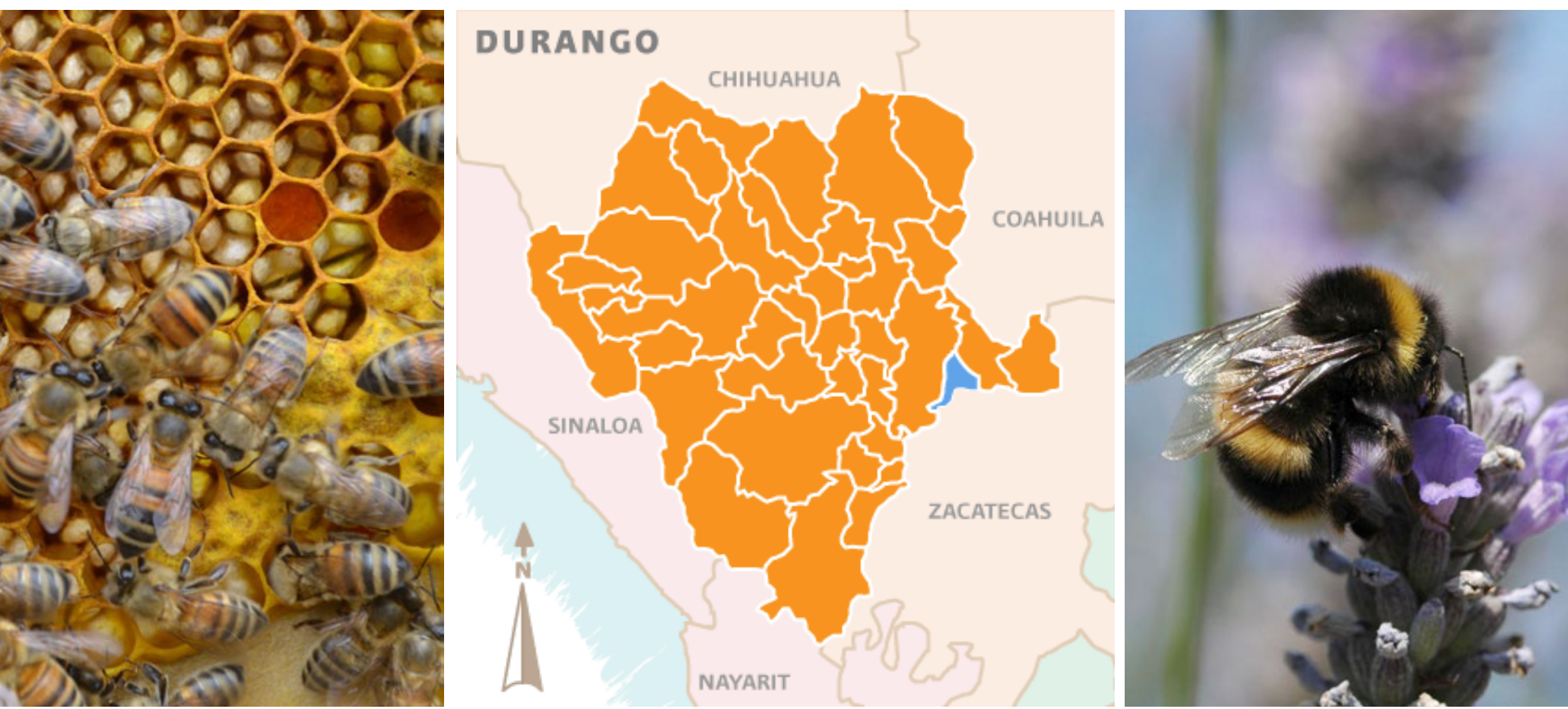

1.- Listado de la Flora de importancia apícola del Mezquital, Dgo., Méx

\section{ACANTHACEAE}

- Carlowrightia serpyllifolia A.Gray

\section{AMARANTHACEAE}

- Amaranthus hybridus L.

\section{ANACARDIACEAE}

- Rhus virens Lindh. ex A. Gray

- Rhus terebinthifolia Schlecht. \& Cham

\section{APOCYNACEAE}

- Asclepias linaria Cav.

\section{ASPARAGACEAE}

- Agave durangensis Gentry

- Agave vilmoriniana A. Berger

- Agave salmiana Otto ex Salm-Dyck

- Dasylirion durangense Trel. .

- Yucca spp.

\section{BIGNONIACEAE}

- Tecoma stans (L.) Juss. ex Kunth

\section{BRASSICACEAE}

- Brassica nigra (L.) K.Koch

- Brassica rapa L.

- Eruca vesicaria (L.) Cav. subsp. sativa (Mill.) Thell.)

\section{BURSERACEAE}

- Bursera palmeri S.Watson

- Bursera sp.

\section{CACTACEAE}

- Mammillaria sp.

- Myrtillocactus geometrizans

(Mart. ex Pfeiff.) Console

- Opuntia sp.

- Stenocereus montanus

(Britton \& Rose) Buxb.

\section{CAMPANULACEAE}

- Lobelia fenestralis Cav.

- Lobelia laxiflora Kunth.

\section{COMPOSITAE}

- Ageratina triniona (McVaugh)

R.M.King \& H.Rob.

- Ambrosia ambrosioides

(Cav.) W.W.Payne

- Ambrosia canescens A.Gray

- Baccharis heterophylla Kunth

- Baccharis pteronioides DC.

- Baccharis salicifolia Nutt

- Berlandiera lyrata Benth.

- Bidens odorata Coville

- Brickellia veronicifolia (Kunth) A.Gray

- Chloracantha spinosa (Benth.) G.L. Nesom

- Flaveria trinervia (Spreng.) C.Mohr

- Melampodium perfoliatum (Cav.) Kunth

- Montanoa leucantha (Lag.) S.F.Blake
- Parthenium bipinnatifidum (Ortega) Rollins

- Pinaropappus roseus (Less.) Less.

- Sanvitalia procumbens Lam.

- Senecio salignus DC.

- Simsia amplexicaulis (Cav.) Pers.

- Tagetes lunulata Ortega

- Taraxacum campylodes G.E.Haglund

- Tithonia tubaeformis (Jacq.) Cass.

- Tridax balbisioides (Kunth) A.Gray

- Trigonospermum annuum McVaugh \& Laskowski

- Trixis angustifolia DC.

- Viguiera dentata (Cav.) Spreng.

- Zaluzania augusta (Lag.) Sch. Bip.

\section{CONVOLVULACEAE}

- Cuscuta mitriformis Engelm.

- Evolvulus alsinoides (L.)

- Ipomoea arborescens (Humb. \& Bonpl. ex Willd.) G. Don

- Ipomoea murucoides Roem. \& Schult.

- Ipomoea purpurea (L.) Roth

- Ipomoea tricolor Cav.

\section{CUCURBITACEAE}

- Sechiopsis tetraptera Dieterle

\section{ERICACEAE}

- Arctostaphylos pungens Kunth 
EUPHORBIACEAE

- Croton flavescens Greenm.

- Croton sp.

- Ditaxis neomexicana

(Müll.Arg.) A.Heller

- Euphorbia antisyphilitica Zucc.

- Euphorbia indivisa

- Euphorbiasp.

- Jatropha dioica Sessé

- Ricinus communis L.

\section{FABACEAE}

- Acacia schaffneri (S.Watson) F.J.Herm.

- Acacia constricta Benth.

- Acacia angustissima (Mill.) Kuntze

- Acacia pennatula

- Calliandra eriophylla Benth.

- Cercidium praecox

(Ruiz \& Pav.) Harms

- Dalea bicolor Humb. \&

Bonpl. ex Willd.

- Desmodium distortum (Aubl.)

J. F. Macbr.

- Desmodium sp

- Eysenhardtia polystachya

(Ortega) Sarg.

- Eysenhardtia sp.

- Lysiloma divaricatum (Jacq.) J.F.Macbr.

- Lonchocarpus sp

- Marina neglecta (Robinson) Barneby

- Medicago sativa L.

- Mimosa monancistra Benth.

- Mimosasp.

- Phaseolus microcarpus Mart.

- Phaseolus vulgaris $\mathrm{L}$.

- Prosopis laevigata (Willd.) M.C.Johnst.

FOUQUIERIACEAE

- Fouquieria splendens Engelm.
HYDROPHYLLACEAE

- Wigandia urens

(Ruiz \& Pavón) Kunth

LAMIACEAE

- Salvia pruinosa Fernald

LOASACEAE

- Cevallia sinuata Lag.

- Gronovia scandens L.

- Mentzelia hispida Willd.

\section{MALVACEAE}

- Melochia tomentosa L.

- Sphaeralcea angustifolia (Cav.) G.Don

- Sida abutilifolia Mill.

- Sida collina Schltdl.

NYCTAGINACEAE

- Mirabilis albida (Walter) Heimerl

- Mirabilis viscosa Cav.

- Boerhavia scandens L.

\section{ONAGRACEAE}

- Ludwigia palustris (L.) Elliott

- Oenothera rosea L'Hér. ex Aiton

\section{OXALIDACEAE}

- Oxalis spp.

PAPAVERACEAE

- Argemone ochroleuca Sweet

POACEAE

- Buchloe dactyloides

(Nutt.) Engelm.

- Cynodon dactylon (L.) Pers.

POLEMONIACEAE

- Loeselia mexicana (Lam.) Brand

- Loeselia scariosa (M. Martens \& Galeotti) Walp.

\section{RANUNCULACEAE}

- Halerpestes cymbalaria (Pursh) Greene

\section{RESEDACEAE}

- Reseda luteola L.

RHAMNACEAE

- Karwinskia humboldtiana (Schult.) Zucc.

ROSACEAE

- Cowania mexicana A.Gray

RUBIACEAE

- Randia sp.

\section{SALICACEAE}

- Populus fremontii S.Watson

- Salix bonplandiana Kunth

- Salix taxifolia Kunth

\section{SAPINDACEAE}

- Dodonaea viscosa (L.) Jacq.

- Sapindus saponaria L.

\section{SCROPHULARIACEAE}

- Buddleja cordata Kunth

- Buddleja sessiliflora Kunth

- Maurandya antirrhiniflora Humb. \& Bonpl. ex Willd.

SOLANACEAE

- Datura sp.

- Nicotiana glauca Graham

- Solanum elaeagnifolium Cav.

- Solanum rostratum Dunal

\section{ULMACEAE}

- Celtis pallida Torr.

\section{VERBENACEAE}

- Aloysia gratissima (Gillies \& Hook.) Tronc.

- Lippia graveolens Kunth

- Lippia sp.

- Phyla nodiflora (L.) Greene

- Verbena carolina L.

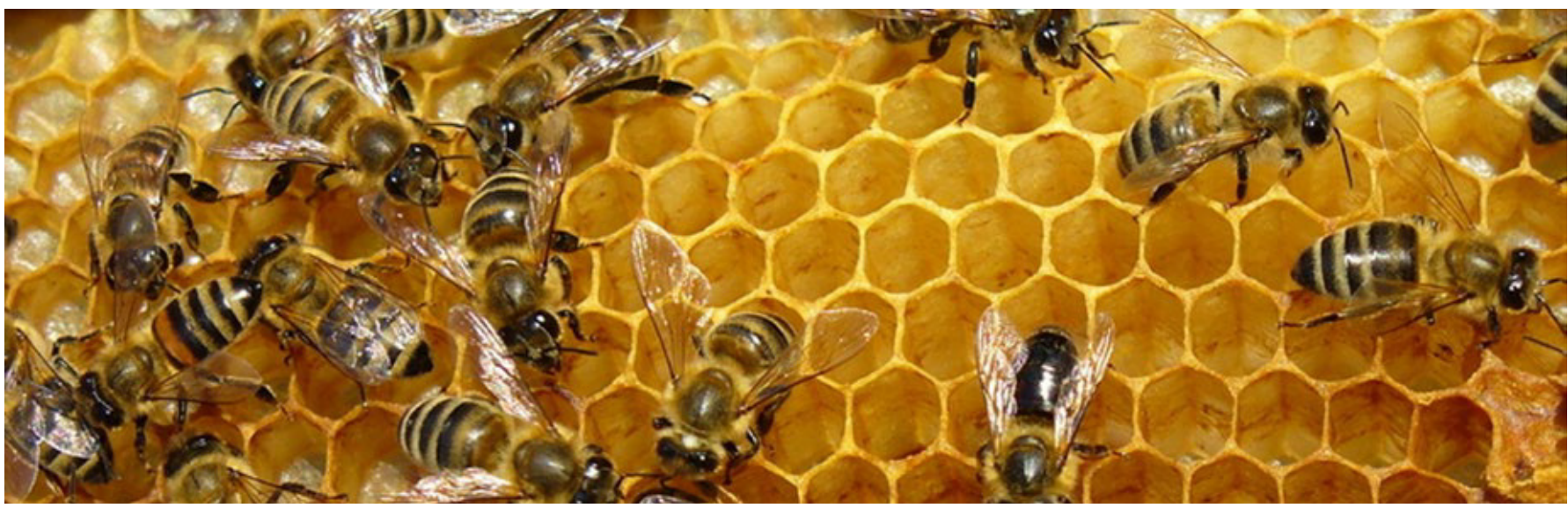




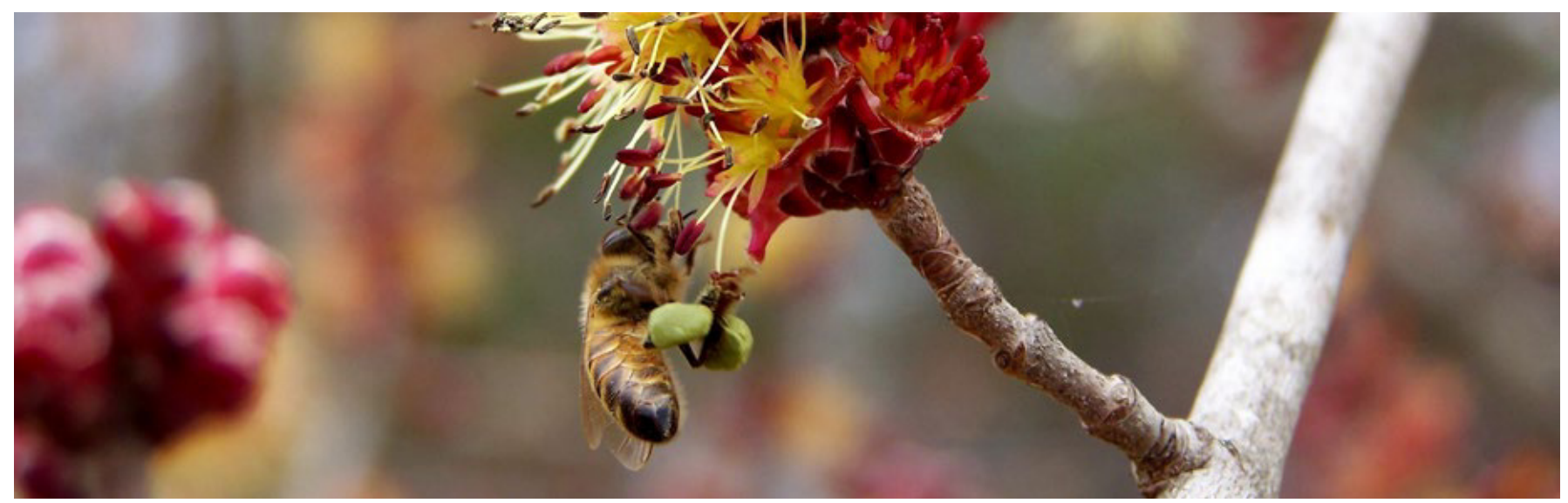

2. Lista de nuevos registros para Durango de especies de importancia apícola.

Agave vilmoriniana A. Berger

Ageratina triniona (McVaugh) R.M.King \& H.Rob.

Baccharis heterophylla Kunth

Buchloe dactyloides (Nutt.) Engelm.

Bursera palmeri S.Watson

Carlowrightia serpyllifolia A.Gray

Cercidium praecox (Ruiz \& Pav.) Harms

Cevallia sinuata Lag.

Cowania mexicana A.Gray

Croton flavescens Greenm.

Chloracantha spinosa (Benth.) G.L. Nesom

Dasylirion durangense Trel

Desmodium distortum (Aubl.) J. F. Macbr.

Ditaxis neomexicana (Müll.Arg.) A.Heller

Dodonaea viscosa (L.) Jacq.

Euphorbia indivisa (Engelm.) Tidestr.

Gronovia scandens L.

Ipomoea murucoides Roem. \& Schult.

Ipomoea tricolor Cav.

Karwinskia humboldtiana (Schult.) Zucc.

Lonchocarpus sp

Marina neglecta (Robinson) Barneby

Melochia tomentosa $L$.

Mirabilis albida (Walter) Heimerl

Mirabilis viscosa Cav.

Phaseolus microcarpus Mart.

Rhus terebinthifolia Schlecht. \& Cham

Salvia pruinosa Fernald

Sapindus saponaria $\mathrm{L}$.

Sechiopsis tetraptera Dieterle

Tecoma stans (L.) Juss. ex Kunth

\section{BIBLIOGRAFÍA}

[1] D. Ramírez Noya. "Exploradores y colectores botánicos de material biológico del siglo XIX en Durango". Alarife 2000 Nueva época. Vol. V; 51. pp. 15-18, 2006.

[2] A.Wulfrath and J. Speck. Enciclopedia Apícola. México, D.F. Agricola Mexicana, 1953.

[3] M. González-Castillo, D. Ramírez-Noya, S. AcostaCastellanos. "Flora utilizada por Apis mellifera L. en la producción de miel en el municipio del Mezquital, Durango". 1st. Cong. Asoc. Mex. Sistm Artrp., 2018, pp.109, CD. MX.

[4] M. González-Castillo, D. Ramírez-Noya, S. AcostaCastellanos. "Análisis palinológico de muestras de miel de Apis mellifera L., provenientes del Mezquital, Durango." 25th. Cong. Int. Act. Apí., 2018, pp. 23-27. Queretaro, Qro., Méx.

[5] M. González-Castillo, M. Rosales Castro; S. Alvarado Aguilar. "Composición química del polen colectado por abejas (Apis mellifera L.), en dos regiones del estado de Durango." 25th. Cong. Int. Act. Apí., 2018, pp. 33-36. Queretaro, Qro., Méx.

[6] E. Hernández, M. Castillo-González, S. Acosta, D. Ramírez. "Representación geográfica de Asteraceae y Fabaceae de importancia apícola en el valle de Poanas y Mezquital, Durango." 30th. Sem. Int. Agrn., 2018, pp. 11691175. Gomez Palacio, Dgo., Méx.

[7] V. Orozco G., M. González Castillo, D. Ramírez. "Resultados preliminares del estudio de árboles y arbustos de importancia para la abeja Apis mellifera L., en el valle del Mezquital, Dgo." 30th. Sem. Int. Agr., 2018, pp. 1046-1051. Gómez P., Dgo., Méx.

[8] (2018) INAFED. Enciclopedia de los Municipio y Delegaciones de México. Accessed Jun. 2018. [on line]. Available: $\quad$ http://inafed.gob.mx/work/enciclopedia/ EMM10durango/municipios/10014a.html

[9] (2018) Wikipedia, Mezquital, Durango. Accessed March. Accessed Jun 2018. [on line]. Available: https:// es.wikipedia.org/wiki/Mezquital(municipio)

[10] M.S. González E., M. González E., M. Márquez L. Vegetación y ecorregiones de Durango. Méx., DF.: Plaza y Valdés, 2007.

[11] (2018) Google Earth Pro. Accessed. May 2017. [on line]. Available: https://earth.google.com/

[12] Instituto Nacional de Estadística Geografía e Informática, (INEGI). "Carta de temperaturas medias anuales, Guadalajara. 1: 1, 000,000." México, DF. 2th. imp. 1984.

[13] A. Lot, F. Chiang (comp.). Manual de Herbario. "Administración y manejo de colecciones, técnicas de recolección y preparación de ejemplares botánicos". México, DF.: UNAM, 1986.

[14] D. Ramirez, N. "Resultados preliminares del estudio de la flora apícola en el municipio de Vicente Guerrero, Dgo.", V Sem. Am. Api., 1991, pp. 91-92. Guadalajara, Jal., Méx.

[15] D. Ramírez N. "Nuevos registros de Compositae de importancia apícola en la flora de Durango y México". Interciencia, 2001. Vol.1; 1, pp.18-21. CIIDIR-IPN-DGO. 2001.

[16] D. Ramírez N., M. González Castillo, S. Acosta C., M. García Ramírez. 2018. "Flora fanerogámica silvestre de Importancia apícola, en dos regiones del municipio de Mezquital, Dgo., Mex." 14Th. Cong. Nal. Rec. Biót. Zon. Árid., 2018, pp. 96-97. Bermejillo, Dgo., Méx. 